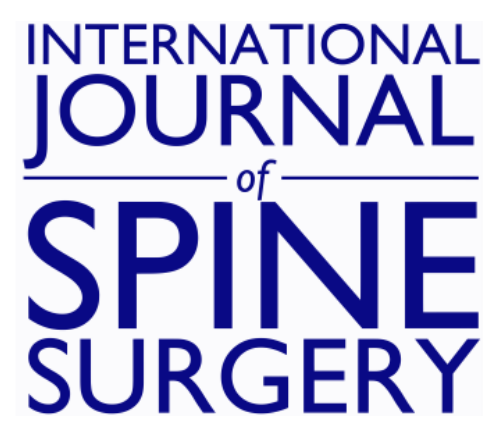

\title{
Basic Science Symposium II: MEMS Technology
}

Shuvo Roy, Aaron Fleischman, Edward C. Benzel, Brent D. Cameron, Lisa Ferrara and Vijay K. Goel

Int J Spine Surg 2008, 2 (2) 120-129

doi: https://doi.org/10.1016/SASJ-2008-Symposium2

http://ijssurgery.com/content/2/2/120

This information is current as of April 26, 2023.

Email Alerts Receive free email-alerts when new articles cite this article. Sign up at: http://ijssurgery.com/alerts 


\section{Basic Science Symposium II: MEMS Technology}

Basic Science Symposia Deputy Editor: Vijay K. Goel, PhD

MEMS Technology Symposium Editor: Lisa Ferrara, PhD

PANEL MEMBERS:

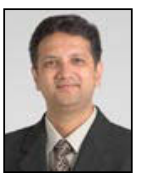

Shuvo Roy, PhD

Department of Biomedical Engineering, Lerner Research Institute,

Cleveland Clinic Foundation

Cleveland, Ohio

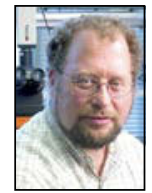

Aaron Fleischman, PhD

BioMEMS Laboratory, Biomedical Engineering, Cleveland Clinic

Cleveland, Ohio

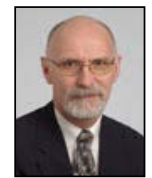

Edward C. Benzel, MD, FACS

Cleveland Clinic Spine Institute

Cleveland, Ohio

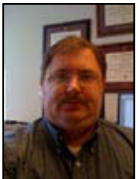

Brent D. Cameron, PhD

Department of Bioengineering, University of Toledo

Toledo, $\mathrm{OH}$

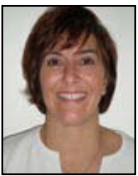

Lisa Ferrara, PhD

OrthoKinetic Technologies, LLC

Southport, NC

\section{INTRODUCTION}

Vijay K. Goel, $P h D^{a}$

For a long time surgeons and basic scientists have been working together in developing instrumentation to restore function across a diseased human joint. In the quest to achieve their objectives during this long journey, they have developed analytical and experimental methods to quantify various parameters across a normal and a diseased joint, like the hip joint. For example, back in the late $80 \mathrm{~s}$ and early $90 \mathrm{~s},{ }^{1-3}$ using crude (from today's perspective) sensor technology, a number of researchers quantified the forces across the hip after total hip arthroplasty. The findings helped the joint replacement industry in several ways including validating the analytical models, contributing to the design of implants that will last longer (hopefully for the life span of the patient), and establishing guidelines for exercise in the immediate postoperative time period. All these results have led to hip arthroplasty improvements to the point that it has become the gold standard for all other joint replacement systems. It is not a surprise that spine surgeons and engineers, with the exponential growth of the bioMEMS technology in the last 5 years, are looking into ways this technology can be used in the area of spine.

Dr. Lisa Ferrara, co-organizer of this symposium, has posed questions ranging from the basic definition of bioMEMS to future/potential applications of the technology in the area of spine. The eminent panel members have provided thought-provoking responses reflecting their experiences in the area. We are fortunate that they agreed to take part in this initiative, and we thank them for their participation. I hope you will enjoy this symposium. We look forward to your comments and submission of your full length manuscripts for the coming issues of the journal.

Key Words: MEMS, spine, smart systems, sensors. SAS Journal. Spring 2008. 2:120-129. DOI: SASJ-2008-Symposium2

${ }^{a}$ Departments of Bioengineering and Orthopaedic Surgery, Colleges of Engineering and Medicine, University of Toledo, Toledo, $\mathrm{OH}$

Address correspondence to Vijay K. Goel, PhD, 5046 NI, MS 303, College of Engineering, University of Toledo, Toledo, OH 43606 (e-mail: Vijay.Goel@utoledo.edu) 


\section{Question \#1. What is MEMS Technology?}

Roy: MEMS (microelectromechanical systems) are devices that involve integrated microdevices or systems, usually comprised of electrical and mechanical components, produced using microelectronics-compatible batch-processing techniques. These systems merge computation with sensing and actuation to perceive the physical world at a miniaturized level.

MEMS technology leverages the infrastructure that was used in the production of electronics chips. And as we are utilizing the infrastructure employed to make electronics chips (such as those used in a computer or an MP3 player), we take advantage of the making of electronics with micromechanical components, which allows us to effectively make smart machines. Also, using the analogy from electronics, our systems are becoming much more sophisticated as we progress in time, and they tend to get smaller (like the iPod basically demonstrates), and at a relatively low cost because we are using batch fabrication. So use of electronics allows us to make tiny circuits at low unit costs. In MEMS technology, we are applying that paradigm to making little mechanical devices - micromachines-which is another name for MEMS. By the way, "MEMS" is the

Fleischman: MEMS stands for microelectromechanical systems, also known as micromachines, and the technology integrates mechanical elements, sensors, actuators, and electronics typically onto silicon substrates. Any tool or measurement technique can conceivably be implemented on microelectromechanical system technology. As a reminder, in the broader sense, MEMS uses term we tend to use in this country; in other places the terms micromachines and microsystems are used. Depending on the audience, these terms may be used interchangeably.

So in the MEMS field, we are trying to make tiny machines at low unit cost, and by putting in electronics, we can make them smart. Over the last 15 years or so we've moved away from just mechanical components; now it can be micro-optical or microfluidic components. It's evolved to a point where MEMS is now applied to almost anything where the technology used to make electronics is used to make something besides electronics.

An example would be a "lab on a chip"—a little biochemistry kit that may be used in a pathology lab. You may be using the same technology employed to make an electronics chip, but here you put in a little sample of, say, blood, and you add a little voltage, and it analyzes the blood chemistry. And there are no mechanical components moving in the system-it's all being driven by fluidics. All this is now called MEMS. It doesn't have to be strictly an electromechanical device anymore. (For further information see Roy et al. ${ }^{4}$ )
Benzel: MEMS technology is a technology that involves the employment of chips like you might utilize from the computer industry but with either moving or functional components. This technology can be used to measure various clinical, biomechanical, or physiological variables, such as the same techniques that the microelectronics industry uses for computer chips. And typically, we can make a MEMS device such that when produced in production quantities not only does it have the advantages of high accuracy and precise dimensional control, but it can typically be much cheaper than conventional devices that are macromachines assembled by hand.
Cameron: MEMS is an acronym that stands for microelectromechanical systems. It is the integration of mechanical elements, sensors, actuators, and electronics on a substrate such as silicon, in which micro-fabrication technologies are used. These fabrication technologies differ depending on what you want to accomplish. If you're trying to incorporate electronics, they are normally fabricated using standard integrated circuit processes or sequences, such as Complementary Metal-Oxide Semiconductor (CMOS) technology or bipolar technology. On the mechanical side, the micromechanical components are fabricated using compatible micromachining processes that in essence selectively etch away parts of the silicon wafer or add new structural layers to form a mechanical device, or an electromechanical device if it has additional integrated electronics.

There are several types of methods used for micromechanical fabrications. Two of the common techniques are MUMPS, pressure or strain. And in particular with the spine, the latter 2 measurements are of utmost importance. We could, perhaps, use such information to establish the presence or absence of bone healing or to assess intradiscal pressure, etc.

which applies to a poly-silicon and stands for Multi-User MEMS Processing System. A similar process called MUSIC is used for silicon carbide, another common substrate material used in MEMS.

Through MEMS it is possible to incorporate micro-scale types of devices such as motors, pumps, fluidic channels, sample preparation (including mixing or vaporization) chambers, and various types of sensors (including optical sensors) that will perform assorted tasks, such as monitoring. Again, you can do both monitoring in the physical sense and the chemical sense. Therefore, MEMS can be thought of as an enabling technology that allows for the development of what we commonly call smart or intelligent systems, which operate without the need for external computing resources. These integrated microelectronics can process the information derived from the sensors and through some decision-making process direct actuators to respond by moving, positioning, regulating, 
pumping, and/or filtering, thereby controlling the environment for some desired outcome or purpose.

Because MEMS devices are manufactured using batch fabrication techniques similar to those used over multiple decades in the integrated circuit industry, we see unprecedented levels of functionality, reliability, and sophistication being placed on small silicon chips at a relatively low cost; so again there is significant potential for MEMS technologies.
Ferrara: MEMS technology is an abbreviation for microelectromechanical system technology (MEMS) that uses integrated circuit fabrication processing and combines it with micromachining techniques to produce micro-sized devices that exhibit high resolution, electronic accuracy, miniature sizing, and have the capacity to monitor long-term, real-time mechanical, chemical, thermal, or electrical parameters for a variety of situations. Utilizing this technology in medical applications would allow in vivo measurements of parameters such as pressures, forces, and strains for long-term applications that require monitoring the condition and status of human tissues and their interactions with medical treatments, surgical stabilization methods, and implantation. (For additional information see Roy et al. and Ferrara et al.$^{6-9}$ )

\section{Question \#2. What are the potential applications in the spine industry for MEMS technology?}

Roy: Because MEMS is a toolkit for making things, we can utilize it for making things that are used inside the patient, things that are employed by the doctor to do things to the patient, or things the doctor will use to learn about patient conditions. By this last one, I mean MEMS can be a research tool that the doctor could use to learn about the spine.

Let me give you an example of some of the different potential applications. One of the applications of MEMS technology is to make sensors that measure certain parameters. A typical MEMS sensor is measuring pressure - a pressure sensor made using MEMS technology is a little tiny chip, about one-tenth of an inch or smaller, and it's very low cost. You could use this sensor to monitor pressure, and depending on how you configure it, you could use it to measure strains, stress on joints, on implants, and other tissues. It could be used to monitor biomechanical loads within tissue either during surgery, after surgery, or even prior to surgery to determine if the loads are pathological.

So as an example of MEMS use in spine medicine, let's say you have someone with a suspected bad disc, ie, disc degeneration. There is already evidence from the work done early on in the 1960s by Nachemson and his colleagues that disc degeneration usually manifests itself with lowered pressures in the nucleus of the disc. So if a spine specialist wanted to know how bad a patient's disc degeneration is, the tiny pressure sensor inserted into nucleus performs as a minimally invasive tool to help determine the state of degeneration. That's a physical parameter. But you might also want to measure the levels of chemicals that are associated with disc degeneration. So you could have a chemical sensor that measures the breakdown of collagen in tissues. MEMS can be used to make physical sensors or chemical sensors. And chemical sensors in general tend to be used for short periods of time, while physical sensors can be used for short periods of time or longer periods of time.

You could also now imagine that one of these load sensors (that is measuring some sort of strain, stress, pressure) could be mounted on an implant. Think of a spine plate, for example, where the spine plate is put into the patient, but then maybe the fusion process is not going well or there's too much undesirable motion, causing little changes in the biomechanical load parameter that will be determined by the sensor. So by monitoring the sensor (mounted on the plate) readings, you'll be able to tell that the plate is seeing the optimum load, and the pattern of fluctuations that will be indicated are of failure versus non-failure.

Another application of MEMS technology is to exploit its precision patterning capabilities. We use techniques like photolithography (which allows for very fine patterning) to create little scaffolds in polymeric materials that you could use to guide stem cells and deposit extracellular matrix (ECM). So you could actually develop tissue engineering scaffolds using these precision patterns. There is some research evidence out there that demonstrates that certain types of patterns accelerate the growth and definition of ECM, so this would be a way, for example, to regenerate the disc.

Another application is in bone healing. After doing a bone graft, you hope the bone graft fuses, but if you don't give it the proper osteoconductive environment, it will not work out. By using MEMS technology to direct appropriate cells on a scaffold that has been patterned with these optimized textures, you'd actually improve the likelihood of bone healing and tissue regrowth.

MEMS technology could be used in making little micromachines that could not only do sensing but could accomplish something else like pumping a drug. So effectively you could make a little drug delivery pump, like a pump system for delivering insulin for diabetic patients, but as a smaller version that is implantable or wearable but would deliver osteobiologics to a region or a tissue. And if it has integrated sensors, it could determine when this should be delivered based on what the sensing parameter is. Say you want to deliver to a patient a specific dosage at a specific time over a period of 7 days. By integrating all this with electronic circuitry, you could program the onboard computer to deliver the drug, and a little micromachine pump would deliver the drug at the appropriate times. 
One more application is the development of what I call smart surgical tools. We currently have all kinds of tools to do cutting and repair of tissue. How about if you integrate sensors so that you are able to tell the difference between bone, muscle, ligament, tendon, and blood vessel? You'd have much more precision cutting and carpentry of the surgical site because now you might have a smart scalpel, an instrument with a MEMS sensor. For example, say you are cutting tissue or you are clearing up the area, and you come too close to a blood vessel. The instrument would then basically trigger a warning light or send a little buzz warning not to go any closer, or you'll reach the aorta or the spinal cord. So by attaching these sensors and working out a little algorithm using tissue information - cutting soft tissue is different than cutting hard tissue-you could measure the forces, and you'd be able to distinguish between the different kinds of tissue. This by itself may be helpful, but this tool would be even more helpful in minimally invasive surgery which is done with minimal hand access. You're submitting a scalpel through a little incision and the scalpel is controlled via image-guided surgery, and with a sensor it could alert you that you are too close. Alternatively you could make a little actuator, such as a vibrating tool on the cutting surfaces, so you could achieve better precision cutting.

So MEMS is not one thing; it is like a toolkit to make things. The key is to take advantage of what I call (1) miniaturization, making things small and with precision; (2) multiplicity, making many things at one time so you can have relatively low unit cost (based on the semiconductor and electronics production industry); and (3) microelectronics, making things with electronics so they are effectively smart devices. That's what I call the 3 Ms of MEMS: miniaturization, multiplicity and microelectronics. (For further information see Benzel et al..$^{5}$ )

(2) in situ surgical evaluation of placement of spinal fixturing, and post-surgical follow-up for the evaluation of the success of surgical intervention, and (3) the application of physical therapy and exercise modification of direct feedback to the course of physical therapy typically required. application areas are primarily grouped into three main areas with, I suspect, a significant amount of overlap: (1) diagnostic,

Benzel: As already alluded to, the primary applications regarding spine care would likely be for the assessment of intradiscal pressure and for the assessment of bone healing. The assessment of intradiscal pressure may demonstrate information that correlates with the size of the neutral zone and, hence, the "stability" of a motion segment. The pressure and patterns of fluctuation of intradiscal pressure may indeed be indicative of both normal and pathological motion segment conditions.

Cameron: In general, MEMS has the potential to revolutionize nearly every product category by bringing together siliconbased microelectronics with micromachining technology. You hear this expression used quite often: "MEMS can be used to create systems on a chip." In a broader sense, when we talk about MEMS, we talk about these "systems on a chip" where we take different functionalities and integrate them under one umbrella.

In regards to the spine, there are also numerous applications related to research, development, and commercial products. Our group and others have been working on incorporating MEMStype sensors directly into intervertebral spinal disc implants. This is done to monitor the mechanical conditions of the implant in vivo. Once it is placed in the body, however, we need some method of getting the collected information out of the body, and it is preferable to avoid invasive approaches (eg, where there are wires coming out of the body). Therefore, in our research, we use a radio frequency (RF) approach. To achieve this, a very micro RF transceiver is fabricated within the implant to couple the information wirelessly out of the body.
Bone healing can be assessed by measuring pressure within bone (at bone/bone interfaces) or between bone and a plate (bone/ implant interfaces) under loading and unloading conditions. Fluctuations in pressure between bone and a plate, for example, are suggestive of undesirable motion or instability and suggest that bone healing may not have transpired. Conversely, a constant pressure between a bone and plate under loading and unloading conditions, for example, should suggest that there is stability and probable healing of a fusion.

It should also be mentioned that such integrated technology has multiple applications. The collected information could be used to improve future implant design. With these sensors integrated throughout the implant, high stress points could be identified, as well as premature wear and potential failure points. Although such information, in the past, has been gathered through lab or bench testing, those types of tests are limited. There are some things that cannot be easily identified or simulated in lab or bench tests, such as how the implant will respond in a realworld environment over extended time duration. Therefore, acquiring in vivo data with the implant experiencing realistic forces and movements could definitely lead to better and more robust implant designs.

As another application, this technology could provide useful clinical information for physicians. Once a MEMS-based implant is in place, it would be much easier to identify potential problems, before possible irreversible damage to the implant is caused, and thus guarantee maximum implant longevity. For example, if someone has an implant and is doing something in his/her everyday life that may eventually cause the device 
to fail, these electronics and computing resources could be logging the events. The physician would then be able to advise the patient that he or she is doing something in everyday life (at a specific time) that may damage the implant if the activity is continued. Therefore, such information could be used to provide feedback to the patient on how he or she may want to adapt their lifestyle.
Ferrara: Physical, chemical, and mechanical parameters can be monitored in vivo to follow a patient's condition and provide treatment strategies that address many stages of a patient's back pain. With respect to spinal implantation, a patient's degenerative status can be monitored early on and through the degenerative process, providing an improved ability to strategize and customize the treatment course for each patient.
Furthermore, the recent surge of novel technologies related to spinal arthrodesis and arthroplasty have posed some challenging mechanical environments. Ideally, implant integrity could be monitored using parameters such as pressure and strain prior to the point of extreme failure that would require explantation of spinal implantation. (For additional information see Ferrara et al. ${ }^{10}$ )

\section{Question \#3. What are the challenges and pitfalls with implantable MEMS technology?}

Roy: Implantable MEMS brings two issues to the forefront. You are going to leave this material in the body for a long period of time and most of the material that these devices are made out of is not a traditional biomedical material; it is not titanium, not stainless steel, not the polymers that everyone knows. That's because we are taking advantage of the capabilities of the electronics industry, and they have perfected making smart devices out of MEMS using silicon. So the key question that comes up is how is this material going to behave in the body? If you're going to leave something in for a long time, how is the material going to affect the body, but also how does the body affect the material? Will the performance change?

Say you have a load sensor on an implant and it is being corroded away. The readout it is going to give is different than if it were not corroding. The material of the sensor is being eaten away by the body fluids. The harsh environment of the body makes biocompatibility of MEMS materials an issue for implantable devices. Biocompatibility is something that we will all have to address in rigorous manner for long-term implantations.

In our group, we have worked on site-specific biocompatibility of MEMS in the spine. We have done in vitro tests on the bench to qualify materials used in the construction of MEMS devices. And we have done some long-term animal tests to investigate in vivo performance and tissue reaction.

The second challenge is telemetry, especially for smart devices that are going to communicate the measurement parameter, whether it is strain or collagen breakdown, whatever it is, from within the body. You have this little chip (think of it as the size of a grain of rice or smaller) that is mounted onto this implant, the disc or bone graft, and you're trying to read its parameters - what its output is. We're communicating with that chip through electronic telemetry, particularly wireless telemetry for implants. That typically requires power. Power usually requires batteries. Unfortunately, batteries also mean large size. If you think in terms of the pacemaker, the largest component of the pacemaker is the battery. But if you are going to put a large component into the disc or on implants, there are
2 problems. You're taking away the inherent size advantageMEMS devices are supposed to be small; and now you're putting in a huge battery next to it. The other disadvantage of a battery is that batteries die eventually. Pacemakers don't really require a lot of power, so you survive. But typical electromechanical sensors based on MEMS consume more power. So the battery dies, and the device stops working.

Another question that comes up is that many of the materials that make up the battery tend to be highly toxic. And the way they get around it in the pacemaker is to put it into this hermetically sealed can, which again can detract from the miniature size needs. I think if you're going to make MEMS universally applicable to spine conditions (and we're proposing to apply them to a number of things like monitoring implant integrity and monitoring biomechanical parameters), we have to eliminate the issue of large size.

We have to come up with technologies to resolve these questions that arise with wireless telemetry and communication in the implanted device. So we have to come up with new ways to address signal transmission and reception. And if you are to fix that, you also want to be able to do it without a battery, if possible. You also want to do it where you don't have too large a size.

So these are the big challenges with implantable MEMS technology: (1) biocompatibility-how does the material behave and how does the body affect the material; and (2) the telemetry between the sensor/actuator and the outside.

Another challenge/pitfall (not a technical one) is the fact that the people who have traditionally developed MEMS technology tend to be mechanical, electrical, and chemical engineers. When we're talking about spine applications, we need to have spine specialists, whether they are surgeons or therapists or basic scientists. One key challenge is that there is really no forum for bringing these two communities together. So, this Journal's symposium is actually a very good, and probably a unique, platform. 
Fleischman: I don't think there are any challenges or pitfalls that are unique to implantable MEMS technologies. Typically, the same concerns that apply to any other implants apply to MEMS implants. If anything, I suspect that it is likely to be a lot easier to have MEMS technologies implemented. And one of the things MEMS devices have in common is they tend to be very, very small. Typically I would envision MEMS devices being mounted on previously existing fixturing or being so small as to have nearly negligible impact on the patients.
I should mention, the typical challenges we are concerned about are still: (1) biocompatibility, (2) in cases where we're going to use MEMS on a telemetry basis, having adequate signal to noise transmitted from the MEMS device, and (3) protecting the MEMS device from the fairly aggressive biological responses that are normal for typical implants.
Benzel: The challenges are related to design and implementation, as well as the provision of an appreciation of the utility of the measured pressure, strain, and load assessments. Other pitfalls may be related to the misinterpretation of the information provided.

Cameron: One of the main challenges with an implanted MEMS technology is its long-term stability once implanted into the body. With it understood that some of these implants may be in the body for 10 to 20 years, they must be able to withstand the body's harsh environment, in both a chemical as well as physical sense. This must especially be considered in the spine, where the loads are tremendous and the potential for wear is severe. Unlike tissue that has the capability for repair, the problem is considerably more complex from a device standpoint. If an implant is going to be placed into the body, one must consider how the device is going to perform over the long term. This is especially important for integrated MEMStype implants with a high degree of system integration (eg, multiple devices on a MEMS sensor platform). If the MEMS device has pumps, fluidic channels, and/or electronics, often a single failure in any one of these subsystems may render the whole device useless, which must always be considered from a design standpoint.

Ferrara: There are biocompatibility challenges with MEMS materials exposed in the harsh fluid environment of the human body. However, numerous and promising coating and surface technologies have been investigated for the long-term
Finally, as data is serially accumulated, much more will be learned about the nuances related to the clinical significance of the acquired information. As this information is collected, the pitfalls should be defined and neutralized and the advantages should become more evident.

As you think about the individual things that can be incorporated on MEMS devices, such as in the integration of chemical sensors to monitor the implant environment, some additional considerations include: When the chemical sensor is reagent-based there must be thought about the consumables, eg, if needed, how can the reagent be replenished? How many measurements can you take over a certain time period? Is the reagent subject to degradation? Furthermore, if a membrane or film is utilized in the sensing technology; what about the potential for biofouling of the film or membrane? These are all issues that must be considered when placing long-term sensors in the body. Short term, the sensor may work great; however, after a period of months and years, it is quite probable that the membrane may fail or there may be reagent issues that will cause the sensor to become nonfunctional.

implantation of MEMS devices into living tissue. In addition, the incorporation of telemetric means for data transmission provides challenging electrical and communication issues.

Question \#4. If MEMS is incorporated into a spinal implant, what would be the benefits? What are the possible clinical complications?

Roy: A benefit is freedom from expensive imaging tools and potentially misleading artifacts. With a spine implant, one way of monitoring is through performance imaging, usually with X-ray, and sometimes with MRI. MEMS solves some of the disadvantages with traditional imaging. With imaging, you have to bring the patient to the imaging suite, take the images, and then the patient goes home. Then at a later time, the spine specialist looks at the images and makes some judgment calls or diagnosis. With MEMS, you do not need the imaging suite, as the device is already in the patient. You come with the external reading unit that gives you an immediate readout. Or you tell the patient to do an exercise and you read out how the measurements change. So you're getting real-time readings based on the patient's daily activities.

You could also instrument the patient with a little belt that has a reader unit, and this reader unit would continuously interrogate the sensor of the MEMS device so you know exactly how this implant is performing over time. Let's say the patient has had a spine implant and has to go up and down stairs. The patient is doing better, but your reading is telling you the implant is loosening. You could have this information recorded in the reader unit that the patient is wearing in the belt, and you could give it to the doctor. Or the patient could look at the unit which 
could be equipped with a red light, yellow light, green light mechanism, and the patient could decide to take it easy on his daily activities based on the readings.

So a big benefit is obtaining real-time, extended or even continuous information to assess implant integrity. As an example of what I mean by extended information, you could have the patient do a number of exercises, and you would obtain measurements and their fluctuations. Or by continuous, I would use the example of the patient who wears an instrumented belt that reads the sensor continuously and provides updates. And the belt could have a little circuit board with a small computer that had green/yellow/red lights, and would warn via a red light that you must go see the surgeon.

Imaging is sometimes misleading. There are cases where it may look like a patient has fused after spine fusion surgery or a patient is doing well after implantation of a motion-

Fleischman: As a caveat, I'm not a clinician; I don't see any clinical complications from having a MEMS device incorporated into a spinal implant. It would be difficult to imagine a case where an extremely small MEMS device, perhaps only a square millimeter, would have any significant

Benzel: To answer the second question first, the clinical complications should be nil unless misinterpretation of the information is considered a variable here. The benefits are inextricably related to the acquisition of information that provides clinically related and clinically useful information,

Cameron: Many of the benefits of incorporating MEMS in spinal applications have already been mentioned in the discussion of the general benefits of such technologies as well as some of the possible clinical complications.

From a more mechanical standpoint that may lead to a clinical complication, if you take a spinal implant and integrate MEMS sensors within or onto the implant material, you must consider how this integration will affect the material itself. Through the

Ferrara: Currently, conventional methods to examine tissue healing and bony incorporation include radiographic evaluation of the tissue, MRI, CT, and the patient's history. Yet, discrepancies exist between radiographic evidence where cited studies demonstrated that plain radiographs have often preservation device. But if the imaging is inaccurate; there are still problems. Therefore, MEMS could be an adjunct to imaging. For example, when imaging says things are okay but the sensors say things are not okay, it might be appropriate for the doctor to check on the patient and select appropriate follow-up therapy.

One possible clinical complication in addition to the biocompatibility issues is how you are communicating with the sensor. If you are communicating with the sensor using radio frequency (one possibility), it may be affected by the metal in the implant. So you have to address that.

Another possible complication (although probably not a clinical complication): people may not want to be compliant with wearing the devices or responding to cues.

impact on the structural soundness of any type of implant. I think the worst-case scenario would be if the MEMS device failed, but then we're simply no worse off than we currently are with our passive, non-smart implants.

such as bone healing status information that assists in the clinical decision-making process. Since this has great potential to be clinically useful information to the clinician, the pitfalls should be far outweighed by the benefits.

act of integrating these sensors, points of stress concentration may be introduced into the implant, which may degrade the lifetime of the implant further in comparison to if the sensors were not present. With this said, it's not that such effects cannot be overcome. However, these are just added layers of complexity that must be addressed from a design standpoint. The use of numerical methods, such as Finite Element Analysis (FEA) techniques, has proved quite useful in dealing with such issues.

led to underestimation (by as much as $20 \%$ ) of fusion status. Radiographs can only provide information related to tissue status during a short snapshot in time. Implantable MEMS devices could provide continuous, accurate, real-time means for monitoring the status of tissue or spinal implant integrity.

\section{Question \#5. Can these devices be formed into arrays/grids and if so would they be able to provide stress or pressure maps of bone or soft tissue areas?}

Roy: Yes, the technology is amenable and the economics of batch fabrication make it practically feasible. By taking advantage of the economies of scale that are used in making electronics and using precision patterning, you could make a

Fleischman: One of the beautiful aspects of MEMS devices is that typically when in production they are cheap enough so little grid of stress sensors that are tiny enough that you can get a much finer resolution of stress changes than you would with individual discreet sensors. They could be used to get pressure or stress maps.

that a multiplicity of these small devices can be used to map pressure gradients and stress gradients, and it is conceivable 
that one could outfit an entire region of the spine with a complete pressure distribution sensing system in order to understand what's happening at the surgical levels and the immediate adjacent levels above and below the surgical site.
Benzel: These devices most certainly can be formed into arrays and grids. There may be significant advantages to acquiring information from different points in 3-dimensional space. These include varying points in the region of a fracture or varying points within a disc interspace that could conceivably provide much more information than that acquired by the assessment from a single point alone. Therefore, such a grid approach can provide a significant and graded advantage, in most circumstances, over a single measurement approach.
Cameron: Absolutely, due to the micro-scale nature of MEMS itself, it is very well suited for the fabrication of sensing array structures. When you think about incorporating adjacent data storage and processing with integrated circuit technology there is considerable potential for the type of information that can be acquired. With that said, it is more difficult to fabricate these array-type structures on non-flat or non-planar surfaces. Even if the array elements are to be spread out over a large surface area, often interconnects between the sensors or microelectronics still need to be made which can pose some problems. However, there are some alternatives that can be done as well. One common alternative is to assemble the MEMS elements on a flexible substrate, such as a Kapton strip (DuPont, Wilmington, Delaware). The sensors can be mounted onto these flexible substrates in various geometries that one can basically mount on a multitude of different surface types. However, if the implant is going to experience significant wear then it must be understood that if the substrate is fixated on the surface of the implant, it will also experience significant wear. Therefore, this is an area where considerable MEMS research is being focused.
Also, new fabrication techniques are being explored other than those discussed in Question \#1. One of the new fabrication techniques includes focused ion beam (FIB) technology, in which a projection can be used to structure material on a microor nanometer scale directly on or in the implant material. The nice thing about such techniques is they do not rely on resist materials for patterning and are better suited for the cases we just talked about in terms of awkward geometries, or if you have to spread sensors out across a large area and are worried about the interconnects. In this case, you are literally making the implant itself the sensor or MEMS device.

These are some of the new areas that are emerging which are being driven by demand. As people start thinking about ideas and applications, obvious pitfalls and problems with current technologies are identified. New technologies, such as FIB fabrication, are emerging and the field is developing. In the case of the spine, it will depend on the demand, need, and what people want to accomplish.
Ferrara: Yes, the technology is ideal for forming arrays and/or grids to map large areas of tissue behavior. The microsize nature of a MEMS device and economic feasibility for fabrication are favorable for creating large arrays for data collection in vivo.

Question \#6. How are these devices inserted or mounted to a device or into an area?

Roy: There are 2 approaches: (1) during manufacturing, you mount the device directly on the implant or (2) during surgery, you mount them on the implant, or deliver them to the surgical site using custom tools.

In the first approach, the device could be attached to the implant by the manufacturer. It becomes a production component; it is reliable, and the surgeon and patient are provided a data sheet.

Another approach for implants is that you make the MEMS separately, and in the operating room the surgeon decides he or she wants to put the sensor or the actuator or the MEMS device on the implant. So the surgeon would then take the MEMS device from its case, mount it on the implant of his/her choosing, do a calibration, basically resetting to zero so that now you read what the baseline is, and then insert the implant into the spine, and monitor what the readout is.
There are advantages/disadvantages of both approaches. In the first approach - in manufacturing - you are building into the implant independent of surgeon technique; it's reproducible for everybody. An advantage of the second approach: the surgeon can choose which implant to put this device on and where. That could lead to cost savings, plus it could lead to better information for the surgeon, allowing him/her to say, "This is where I want to measure my parameter; this is where I need to make my device work better."

In terms of delivery of implants, the way you do it is to have custom tools that I think will be derived from the minimally invasive tool industry, and you'd use those to deliver your MEMS chip. A lot of these tools will have to be custommade because MEMS traditionally have not been addressed by people who make surgical tools. So I think there will be a thriving area for innovation. 
Fleischman: The particular case would be dictated by the application. Strain devices would have to be mounted using fixturing cements directly onto the spinal implant devices.

Benzel: These devices could be inserted into a disc interspace by way of a needle or trocar such as one that may be used for discography. They may be inserted into bone by a similar mechanism. Sensors could be attached to an implant in the

Cameron: First of all, how you mount or interface the MEMS sensor to the implant depends on the implant material. From my experience, it's easier to interface MEMS technologies into polymer-type materials. The reason is that often the material itself can be cured around the sensor. Even when it needs to be machined, it is relatively simple to fill in the voids to create a suitable interface with similar mechanical properties. For metals such as titanium, chromium, or steel, it's definitely more difficult.

Commonly, the material to which your MEMS sensor is interfaced will need to be milled for the physical insertion of the device. This often is further complicated, as mentioned before, if interconnects need to be made or there are multiple sensors that are spread out. In the case of interconnects

Ferrara: The micro size of these devices would allow injecting the devices through a needle to the target site or directly mounting them to the surface of the implant. In addition,
Intradiscal pressures could be injected using needles or catheters directly into the disc. Potentially, these devices could be incorporated directly into replacement disc materials.

factory. These devices could perhaps assess implant strain or pressure under the implant and between the implant and the bone. Or they could simply be placed between the implant and the bone at the time of surgery.

between the sensors, when working with materials such as a metal, it is often difficult as both the metal and interconnects are conductive. In this case, insulation layers must also be incorporated. Another option, as mentioned previously, is to use flexible substrates, which can then be affixed to the device itself. If the surface is going to be affected by wear, however, this approach is limited.

Newer technologies are being developed to get around some of these mounting issues. For example, the focused ion beam (FIB) approach previously discussed has potential to minimize some of these problems. It will be quite interesting to see where new technologies will take us in 5-10 years. I believe there will be significant breakthroughs and the technology landscape will be quite different compared to today.

catheter-guided placement with image tracking capabilities has been investigated and would allow for visualization of placement.

Question \#7. What type of regulatory and clinical tests are necessary for FDA approval?

Roy: MEMS for medicine is a nascent technology that is very promising - which means there's a lot of excitement, but you also don't have a track record or history with the FDA. So we basically need trailblazers out there who are going to answer questions like: "What is the reproducibility of these devices? How will they perform under different conditions?"

What kind of tests would you need to do? I suspect you would need to do biocompatibility tests that are ISO-based to be accepted by the FDA. You need to do migration testing-you put a chip on an implant or you put a chip by itself on a disc or on a bone graft and you don't want this chip to migrate away from the desired location and expulse and pierce a blood vessel or do something else to the spinal cord.

Benzel: I am not an expert on the FDA approval process, but more than likely, MEMS devices will be approved for use on the basis of the $510 \mathrm{~K}$ approval process. Therefore,

Cameron: This is definitely not my area of expertise, but I do have a little insight. For the most part, it's not too much different than the clinical and regulatory tests for standard implants. If your implant is deemed a Class III device, it will still need to go through the premarket approval process. It should be mentioned, however, that even an existing Class I or II device
So you have to test for biocompatibility, for migration of the device, and you test whether they are subject to fatigue. Traditionally, the material used to construct most MEMS devices-silicon-tends to be very fatigue-resistant, but I think the FDA will want that verified for specific devices via experimental testing. We can learn a lot from a MEMS device that was approved by the FDA 2 or 3 years ago for a different application, in cardiology, for measuring blood pressure. This long-term implant with a pressure sensor is made by a company called CardioMEMS. Seeing what kind of tests it was asked to perform or what tests it submitted would be something we could learn from. So I would say that you have a series of tests for spine implant, and you have a series of tests for biocompatibility, and possibly others based on whatever CardioMEMS did.

the demonstration of safety and of relative clinical value is implied.

(without MEMS integration) would probably be deemed a Class III device once MEMS technology is integrated into the device, thus requiring premarket approval and additional related clinical trials as well as other approval testing. This is because certain chemicals and materials used in MEMS manufacturing could cause biocompatibility complications. .com/ by guest on April 26, 2023 
Furthermore, when incorporating MEMS devices that are made out of certain materials, if they undergo wear, that wear material-even though in the bulk form may not cause a problem-when the particulate wear matter interacts with surrounding tissue, illness or health-related issues may occur.

Furthermore, if you think about it, although most implants are passive devices, if we are talking about integrated MEMS implants, these devices incorporate electrical components. In one of the examples we are currently working on, the implant incorporates a radio frequency transceiver to wirelessly communicate information from inside the body to an external transceiver. If you incorporate such a device into the implant, there are also FDA regulations that must be followed for RF devices, and appropriate and additional clinical testing for this aspect must be included. In addition, the inclusion of the electrical technology will normally require a power source to be included, such as a battery. Often the battery, if integrated into the implant, would not be easily accessible. Therefore,

Ferrara: There are some implantable sensors that have approval and have a track record with the FDA. However, electronic validation studies, migration, device anchoring,
10 years down the road you may have to worry about the battery structure becoming compromised, even if it was originally hermetically sealed. If it is a chemical-based battery, this material may be toxic, and therefore the FDA would be concerned about the probability of such material leaking into the tissue or surrounding area. Such items may require additional levels of regulatory testing for FDA approval.

In conclusion, it's going to be very interesting to see what is going to be out in the commercial market in 10 years. There are companies right now performing clinical trials with MEMSintegrated implants including those for the spine. Some of these products will be in the commercial market in the near term. For comparison, we are in a time frame similar to when pacemakers were introduced and revolutionized the cardiac field. Similar advances in MEMS are now being made with applications to other bio-fields such as orthopedics.

electronic and mechanical durability will need to be assessed through a series of benchtop, cadaveric, and animal tests.

\section{REFERENCES}

1. Bergman G, Graichen F, Rohlmann A. Hip joint loading during walking and running measured in two patients. J Biomech. 1993;26(8): 969-990.

2. Bergman G, Rohlmann A, Graichen F: Hip joint forces during physical therapy after joint replacement. Trans Orthop Res Soc, New Orleans, 1990; 15:2.

3. Davy DT, Kotzar GM, Brown RW, et al. Telemetric force measurements across the hip after total hip arthroplasty. J Bone Joint Surg Am. 1998;70(1): 45-50.

4. Roy S, Ferrara LA, Fleischman AJ, and Benzel EC. Microelectromechanical systems and neurosurgery: a new era in a new millennium. Neurosurgery. 2001;49:779

5. Benzel EC, Kayanja M, Fleischman AJ, and Roy S. Spine biomechanics: fundamentals and future. Clinical Neurosurg. 2006;53:98

6. Roy S, Ferrara LA, Fleischman AJ, and Benzel EC. Microelectromechanical systems and neurosurgery: a new era in a new millennium. Neurosurgery. 2001;49:779-797.
7. Ferrara L, Fleischman A, Benzel E, and Roy S. Silicon dermabrasion tools for skin resurfacing applications. Med Eng Phys. 2003;25(6):483-490.

8. Ferrara L, Fleischman A, Tokogawa D, et al: An in vivo biocompatibility assessment of MEMS materials for spinal fusion monitoring. Biomed Microdevices. 2003;5:297.

9. Ferrara LA, Gordon I, Coquillette M, et al. A preliminary biomechanical evaluation in a simulated spinal fusion model. Laboratory investigation. $J$ Neurosurg Spine. 2007;7(5):542-548.

10. Ferrara LA, Gordon I, Schlenk R, et al. In vivo assessment of bone graft/endplate contact pressure in a caprine interbody pseudarthrosis model: a preliminary biomechanical characterization of the fusion process for the development of a microelectromechanical systems (MEMS) biosensor. SAS Journal. 2008;2:1-8. 